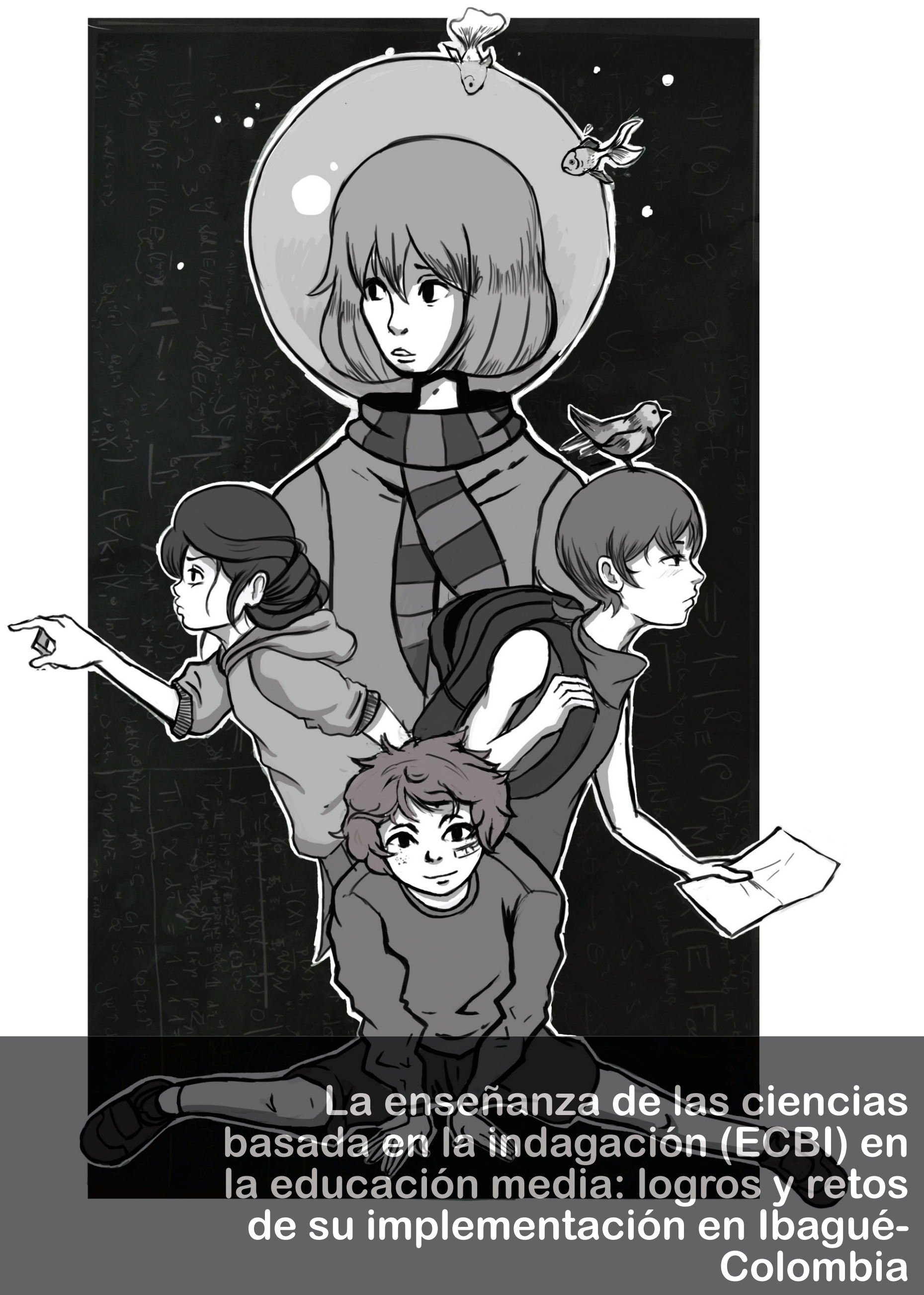




\section{La enseñanza de las ciencias basada en la indagación (ECBI) en la educación media: logros y retos de su implementación en lbagué - Colombia}

Inquiry-based science education (IBSE) in secondary education: achievements and challenges of its implementation in Ibagué, Colombia

\section{Helga Patricia Bermeo (Colombia) \\ Universidad de lbagué \\ helga.bermeo@unibague.edu.co}

Doctora en proyectos de ingeniería e innovación. Profesora e investigadora del grupo GINNOva de la Universidad de Ibagué, Colombia.
0 ensino das ciências baseado na indagação (ECBI) no ensino meio: logros e desafios de sua implementação em Ibagué - Colômbia

\author{
José David Meisel (Colombia) \\ Universidad de los Andes \\ jd.meisel28@uniandes.edu.co
}

Magíster en Ingeniería Industrial. Becario de doctorado,

Facultad de Ingeniería, Universidad de los Andes.

\section{Resumen}

La enseñanza de las ciencias en la población estudiantil joven es una factor clave para que un país cuente con una masa crítica de ciudadanos, alfabetizada científicamente, que facilite y promueva el desarrollo tecnológico, económico y social, bajo un enfoque sustentable. La metodología de enseñanza de las ciencias basada en la indagación (ECBI), se está posicionando internacionalmente como herramienta pedagógica efectiva para generar en los jóvenes el desarrollo de competencias científicas, tecnológicas y ciudadanas. Este artículo se orienta a presentar los detalles de la implementación de la metodología ECBI en un entorno particular: el Programa de Pequeños Científicos (PPC) en la región central del departamento del Tolima, bajo la orientación y gestión de la Universidad de Ibagué. Haciendo uso de los cuestionarios y la entrevista como herramientas de consulta, los resultados derivados del análisis estadístico descriptivo y comparativo de medias, revelan que el perfil institucional, la jornada de clase y el perfil docente parecen ser particulares condiciones que favorecen la implementación del PPC. Este hallazgo alimenta la estrategia de acción para las instituciones gestoras del PPC en Colombia.

\section{Abstract}

Science education for the young school population is a key factor for a country to have a critical mass of science literate citizens that facilitates and promotes technological, social and economical development under a sustainable approach.

Inquiry-Based Science Education (IBSE) methodology is positioning internationally as an effective pedagogical tool to promote the development of scientific, technological and citizen competences in youngsters. This article intends to present the details related to implementation of IBSE methodology in a particular

\section{Resumo}

O ensino das ciências na população estudantil jovem é um fator chave para que um país conte com uma massa crítica de cidadãos, alfabetizada cientificamente, que facilite e promova o desenvolvimento tecnológico, econômico e social, baixo um enfoque sustentável. A metodologia de ensino das ciências baseada na indagação (ECBI), se está posicionando internacionalmente como ferramenta pedagógica efetiva para gerar nos jovens o desenvolvimento de competências científicas, tecnológicas e cidadãs. Este artigo se orienta a apresentar os detalhes da implementação da metodologia ECBI em um 
framework: Little Scientist Program (LSP) in the central region of Tolima, following the guidelines and management of the University of Ibague. Using the survey and interview techniques to collect information, the results derived from descriptive and comparative media analysis reveal that the institutional profile, the class shift, and the teacher's profile seem to be specific conditions which favor the LSP implementation. This finding contributes to the action strategy of the managing institutions of LSP in Colombia.

Palabras clave: $\mathrm{ECBI}$ en Colombia, propuesta pedagógica, Educación en ciencias entorno particular: o Programa de Pequenos Cientistas (PPC) na região central do departamento do Tolima, baixo a orientação e gestão da Universidade de Ibagué. Fazendo uso dos questionários e a entrevista como ferramentas de consulta, os resultados derivados da análise estatística descritiva e comparativa de médias, revelam que o perfil institucional, a jornada de aula e o perfil docente parecem ser particulares condições que favorecem a implementação do PPC. Esta descoberta alimenta a estratégia de ação para as instituições gestoras do PPC na Colômbia

Key words: IBSE in Colombia, pedagogical proposal, science education

Palavras chave: ECBI na Colômbia, proposta pedagógica, Educação em ciências.

\section{Cita recomendada}

Bermeo, H. \& Meisel, J. (2012). La enseñanza de las ciencias basada en la indagación (ECBI) en la educación media: logros y retos de su implementación en Ibagué- Colombia. Panorama, 6(11), 35-45. 


\section{INTRODUCCIÓN}

Para competir con éxito en la sociedad del conocimiento y la tecnología del siglo XXI, se requiere de personas con capacidad para pensar críticamente, resolver problemas y apropiar adecuadamente el uso de tecnologías. En este contexto, toma importancia la necesidad de impartir una educación a lo largo de la vida, que se fundamente en cuatro pilares: 1) aprender a conocer, 2) aprender a hacer, 3) aprender a vivir juntos, 4) aprender a ser (Delors, 1996). En particular, el aprender a hacer es un llamado a ofrecer una educación más que profesionalizante, una educación que capacite a los individuos en la competencia para tomar acción frente a las diversas situaciones que enfrenta en su entorno. Así mismo, con el surgimiento de grandes avances tecnológicos en materia de informática y comunicaciones, es clara la necesidad de que a través de la educación, también se lleve a cabo la alfabetización tecnológica de los ciudadanos, como uno de los factores claves para garantizar el desarrollo sostenible de los pueblos y reducir la brecha tecnológica frente a los países más desarrollados (NSRC, 1997; UNESCO, 2001).

Para lograr lo anterior, se ha subrayado la necesidad de promover una ciencia escolar valida y útil para los estudiantes, en donde el modelo de enseñanza de las ciencias sea coherente y pertinente con la realidad del mundo globalizado, contribuyendo así al desarrollo de una comprensión flexible, sistemática y principalmente crítica del mundo, tanto de los otros como de sí mismos (Acevedo, 2004). Uno de los principales elementos a tener en cuenta, es la importancia que tiene la primera etapa del proceso de educación formal, wn la cual se colocan las bases del pensamiento científico y se promueve la educación por la curiosidad natural en los estudiantes con el fin de desarrollar en ellos hábitos de pensamiento más sistemáticos y autónomos (Furman, 2008).

Los modelos de enseñanza de las ciencias han evolucionado porque día a día se ha buscado el mejoramiento y la optimización del aprendizaje, y porque se ha desarrollado a través de métodos opuestos a la metodología de la enseñanza tradicional; la cual por un lado, privilegia el aprendizaje memorístico y, por otro, el docente es el protagonista en el proceso y finalmente los estudiantes cumplen un rol pasivo en la clase. Es por esto que los nuevos métodos se basan en un enfoque constructivista que propone una construcción conjunta del conocimiento, esto significa que el aprendizaje no es solo un asunto de transmisión, asimilación y acumulación de conocimientos sino un proceso para ensamblar, interpretar y construir conocimiento desde los recursos de la experiencia y la información que recibe el estudiante (Buch \& Wolff, 2000; Barros, 2008).

A partir del enfoque constructivista se han desarrollado dos metodologías principales, los modelos basados en el estudiante como científico y los que vislumbran al estudiante como aprendiz (Barros, 2008). En el primer modelo tiene lugar la metodología de enseñanza por investigación, en donde se utiliza la simulación en clase de las actividades de los grupos de investigación, como principal estrategia para la construcción del conocimiento. En el segundo modelo se afirma que "cuanto más ajustados y precisos se hagan los diseños de enseñanza a los procesos de aprendizaje y desarrollo cognitivo que llevan al estudiante a convertirse en experto en ciencias, mayores serán las posibilidades para provocar esta transformación cognitiva” (Barros, 2008). 
A través de los años se han generado nuevas propuestas metodológicas que complementan y enriquecen las anteriores como la enseñanza de las ciencias basada en indagación conocida por sus siglas iniciales como ECBI (Campanario \& Moya, 1999; Prince \& Felder, 2006; Olier, Duque, \& Tiberio, 2007). Este modelo pedagógico que tuvo sus inicios en los años 70 en los Estados Unidos, bajo el liderazgo del premio Nobel de Física Leon Max Lederman; se basa principalmente en el aprendizaje a través de actividades que implican la realización de observaciones, formulación de preguntas, revisión de fuentes de información y evidencias experimentales, planificación de investigaciones, proposición de respuestas y explicaciones, y comunicación de resultados. La indagación requiere del aprendiz la capacidad para la identificación de supuestos, el uso del pensamiento crítico y lógico, y la consideración de explicaciones alternativas. En este contexto, los estudiantes aprenden la manera científica de conocer el mundo y desarrollan la capacidad de realizar investigaciones (NRC, 2000). Más allá de esto, la ECBI se caracteriza porque exige que los actores inmersos (docentes, estudiantes, comunidad institucional) se cuestionen sobre la ciencia que se debe y es posible enseñar (Campanario \& Moya, 1999). La ECBI obedece a un enfoque multifacético que pretende que los docentes se orienten hacia pedagogías que motiven a los estudiantes al aprendizaje de las ciencias (Deober, 2006).

En Colombia, la ECBI está presente como propuesta pedagógica en el Proyecto Pequeños Científicos (PPC). Este es un programa con el que se busca cambiar la forma de enseñar ciencias en la escuela básica primaria colombiana. Su propósito principal es "desarrollar competencias científicas y tecnológicas, habilidades de comunicación y competencias ciudadanas en su población objetivo” (Duque, 2008). El PPC trabaja directamente con los docentes y las instituciones educativas, e indirectamente con los niños. A los docentes, por medio de un esquema de formación, el PPC les proporciona herramientas básicas para realizar una clase de ciencias basada en la indagación; a las instituciones, el PPC les adelanta un esquema de seguimiento para facilitar la implementación del mismo.

El proceso de evaluación propuesto para el PPC es sistémico e incluye diversas dimensiones de análisis. Dentro de este sistema se identifican dos dimensiones: 1) el sistema de intervención y 2) los resultados de la intervención. Estas dimensiones en su conjunto, responden a los objetivos principales del Proyecto: desarrollo de competencias en los niños, proceso de formación docente, gestión escolar y gestión del proyecto en los núcleos. En particular, los resultados de la intervención se analizan a partir de tres factores principales: cobertura, consolidación y calidad.

Los resultados de la aplicación de este sistema de evaluación del PPC en Colombia, se inician con el trabajo piloto realizado en Bogotá durante el año 2006 (Meisel, 2007; Olier, Duque, \& Tiberio, 2007). Luego, durante el año 2008 se llevó a cabo el proceso de evaluación del Proyecto Pequeños Científicos (PPC) en la ciudad de Ibagué, bajo la administración y coordinación de la Universidad de Ibagué. Particularmente, este artículo aborda el caso de estudio de la implementación del PPC en la ciudad de Ibagué, en donde se analizó el impacto y alcance del programa desde la perspectiva de los estudiantes, teniendo en cuenta factores individuales, grupales e institucionales y su relación con los logros del PPC, en términos de: 1) ambiente de aprendizaje en el aula de clase y 2) el desarrollo de competencias ciudadanas en los estudiantes. 


\section{METODOLOGÍA}

Este artículo se enfoca en el estudio y evaluación del caso de aplicación del PPC en la ciudad de Ibagué, durante el año 2008. Los sujetos de interés fueron los estudiantes de los cursos de $4^{\circ}$ y $5^{\circ}$ primaria de diferentes instituciones educativas de la ciudad de Ibagué. A una muestra conformada por 636 estudiantes, se les aplicó los instrumentos al principio y al final del año de formación en el PPC.

Los factores considerados en los instrumentos de medición incluyeron variables relativas al desarrollo de tres ambientes de aprendizaje: 1) Respeto a la palabra (AMB-RPL), 2) Opinión y participación (AMB-OYP); y 3) Trabajo colaborativo (AMB-TCO). Estos resultados en su valor promedio (calificados originalmente en la escala de 1 a 5) fueron contrastados con la presencia de otras variables de entorno en el marco del PPC, como los siguientes: el perfil institucional PERINT (naturaleza -privada o pública-; tamaño institucional), perfil del curso atendido PERCUR (jornada de trabajo) y perfil docente PERDOC (género, antigüedad en PCC).

De esta forma, se analizaron tres factores como variables independientes y tres factores de resultados como variables dependientespara las fases pre y post de evaluación. Una vez confirmada la validez de los instrumentos de consulta (alfa de Cronbach>0,55) y hecha la reducción de variables (análisis factorial confirmatorio con un 32,9\% de explicación de la información original), se utilizó el análisis estadístico descriptivo e inferencial (Análisis ANOVA y MANOVA) para valorar el valor de cambio logrado en los estudiantes, luego de haber participado durante un año en el PPC, así como para valorar la interacción con las variables de entorno.

\section{RESULTADOS}

El propósito principal de este estudio fue analizar la influencia de factores individuales, grupales e institucionales, en la condición inicial y final de los grupos de estudiantes intervenidos por el PPC en la ciudad de Ibagué. En esta medida, se analizó si existía diferencia en las respuestas de los niños ante los ambientes de aprendizaje de las ciencias, por razón de la presencia de las siguientes variables del entorno: perfil institucional (privada o pública), características del curso, perfil del docente (hombre o mujer) y perfil del estudiante (hombre o mujer).

\section{Análisis de la respuesta a los ambientes de aprendizaje}

Los elementos del ambiente de aprendizaje que se midieron en el salón de clase fueron: Respeto a la palabra, Opinión y Participación; y Trabajo colaborativo. La medida de estas variables en su fase inicial, correspondió a la suma ponderada producto del análisis factorial por componentes principales. Los resultados del análisis descriptivo de estas tres nuevas variables, revelan que en promedio, los niños en su fase inicial muestran que en sus clases hay un buen desarrollo de los elementos más significativos del trabajo cooperativo (valor promedio 4,52); los otros elementos como la opinión y participación y el respeto a la palabra no muestran un alto desarrollo, dado que sólo se presentan algunas veces en sus clases de ciencias (valor promedio 3,63 y 3,57 respectivamente); en la fase post los resultados muestran que en sus clases se mantiene un buen desarrollo de los elementos más significativos 
del trabajo cooperativo (valor promedio 4,46), y los otros elementos como la opinión y participación y el respeto a la palabra no muestran una mejora con respecto a la fase inicial, sólo se presentan algunas veces en sus clases de ciencias (valor promedio 3,52 y 3,64 respectivamente).

\section{El análisis del cambio de respuesta en los ambientes de aprendizaje}

Luego de la intervención del PPC, se analizó a nivel individual y grupal. A nivel de estudiantes, considerando el valor promedio de todas las puntuaciones obtenidas de todos los 636 niños participantes en el PPC, la comparación de medias al inicio y final del Proyecto, revelaron la presencia de un cambio positivo aunque no significativo en los niños para un mayor respeto a la palabra [p-valor (t-test),0,05]. Por otra parte, el análisis a nivel de grupos, considerando el valor promedio de las puntuaciones obtenidas de todos los niños vinculados a un determinado grupo en la fase pre $\&$ post, (según el test de comparación de medias de todos los 24 grupos presentes en el PPC), se reveló un cambio positivo aunque no estadísticamente significativo, en el ambiente vinculado con uno mayor a la palabra [p-valor (t-test),0,05].

\section{Análisis de la interacción de las variables del entorno en el nivel de respuesta obtenido en los ambientes de aprendizaje.}

De acuerdo con lo indicado en la Tabla 1, solo algunas variables del entorno evidencian interacción. La valoración de la incidencia del perfil institucional sobre el desarrollo de ambientes de aprendizaje, los resultados del análisis relacional muestran que este factor hace diferencia en el grado de desarrollo de un ambiente de aprendizaje propicio para el desarrollo de competencias científicas y ciudadanas que se logran inculcar en los estudiantes mediante el PPC. En particular esta situación se evidencia en aquellas instituciones educativas de carácter privado que hacen seguimiento a la implementación del PPC, y cuyo tamaño corresponde a una población estudiantil pequeña (4400 estudiantes por jornada);en éstas, se logra en los participantes del programa PCC una mayor disposición al respeto por la palabra en el aula de clase.

Al evaluar la interacción con el perfil del curso, los resultados muestran que los estudiantes en la jornada de la mañana fueron más afines a trabajar bajo un ambiente de trabajo colaborativo. Finalmente, los resultados relacionados con el perfil docente indican que los estudiantes cuyos cursos fueron orientados por un docente de género masculino y con antigüedad en el proceso de formación bajo la metodología ECBI, presentaron, en general, mayor grado de desarrollo de ambientes de aprendizaje. 
TABla 1. Resultados del análisis ManOVA (P-valor prueba F) para las varlABLES RELATIVAS AL DESARROLLO DEL AMBIENTE DE APRENDIZAJE

\begin{tabular}{clllllll}
\hline \multirow{2}{*}{$\begin{array}{c}\text { Variables de } \\
\text { entorno }\end{array}$} & \multicolumn{1}{c}{ Factores fijos } & AMB_TCO & AMB_OYP & AMB_RPL & AMB_TCO & AMB_OYP & AMB_RPL \\
\cline { 2 - 7 } PER_INT & $\begin{array}{l}\text { Naturaleza jurídica } \\
\text { (Privada) }\end{array}$ & 0,947 & 0,255 & $0,02^{*}$ & 0,770 & $0,03^{*}$ & 0,156 \\
& $\begin{array}{l}\text { Tamaño institucional } \\
\text { (<400 estudiantes) }\end{array}$ & $0,000^{* *}$ & $0,000^{* *}$ & $0,000^{* *}$ & 0,057 & $0,001^{* *}$ & $0,008^{* *}$ \\
\hline PER_CUR & $\begin{array}{l}\text { Jornada de trabajo } \\
\text { (mañana) }\end{array}$ & 0,374 & $0,019^{*}$ & 0,145 & $0,038^{*}$ & 0,591 & 0,161 \\
\hline PER_DOC & $\begin{array}{l}\text { Antigüedad en PPC } \\
\text { (>1 año) }\end{array}$ & $0,008^{* *}$ & $0,000^{* *}$ & 0,350 & 0,066 & $0,021^{*}$ & $0,038^{*}$ \\
& $\begin{array}{l}\text { Género del docente } \\
\text { (Hombre) }\end{array}$ & 0,097 & 0,177 & $0,000^{* *}$ & $0,001^{* *}$ & $0,006^{* *}$ & $0,000^{* *}$ \\
\hline
\end{tabular}

$\left.\left(^{*}\right){ }^{* *}\right)$ Prueba $f$ significativa al 0.05 y 0.01 , respectivamente

\section{CONCLUSIÓN}

Fuente: Datos del Autor.

En esta investigación se evidenció la importancia del factor institucional para el alcance de buenos resultados en el PPC. En particular, se resalta la conveniencia de su implementación en instituciones donde el proceso ya esté consolidado, particularmente, porque existen técnicas de seguimiento y evaluación interna del proceso, así como la disponibilidad de recursos que financian los materiales requeridos para el normal funcionamiento del Proyecto.

Los resultados también sugieren que la presencia de profesores con experiencia en el marco del PPC, así como la orientación del profesor de la jornada de la mañana, son otros dos aspectos que contribuyeron a los logros del Programa. Si bien se identifican cambios de actitud en los niños, estos cambios —una vez medidos antes y después del PPC - no son del todo estadísticamente significativos y no se pueden generalizar en todos los ambientes de aprendizaje propuestos por el Programa.

Desde el punto de vista metodológico, el estudio del PPC en Ibagué deja ver la necesidad de reformular los instrumentos de evaluación para así poder establecer como mayor claridad, los logros del Programa y el proceso de seguimiento a esta metodología más allá del entorno escolar. 


\section{Referencias bibliográficas}

1. Acevedo, J. (2004). Reflexiones sobre las finalidades de las enseñanzas de las ciencias. Revista Eureka, 1, 3-16.

2. Barros, J. (2008). Enseñanza de las ciencias desde una mirada de la didáctica de la escuela francesa. Revista Escuela de Ingeniería de Antioquía (EIA), 10, 55-71.

3. Buch, N., \& Wolff, T. (2000). Classromm teching trough inquiry. Journal of professional issues in engineering education and practice, pp.105 - 109.

4. Campanario, J., \& Moya, A. (1999). ¿Cómo enseñar ciencias? Principales tendencias y propuestas. Enseñanza de las ciencias, 17(2), 179-192.

5. Delors, J. (1996). La educación encierra un tesoro. Informe a la UNESCO de la comisión Internacional sobre la educación para el siglo XXI presidida por Delors. Madrid: Unesco.

6. Deober, G. (2006). Historical perspectives on inquiry teaching schools. En L. Flick \& N. Lederman, Scientific inquiry and nature of science Netherlands. Springer, Dordrecht: Kluwer Academic Publishers

7. Duque, M. (2008). Programa Pequeños Científicos: presentación y alternativas de vinculación. Bogotá: Universidad de los Andes.

8. Furman, M. (2008). Ciencias naturales en la escuela primaria: colocando piedras fundamentales del pensamiento científico. IV Foro Lationamericano de Educación, Aprender y Enseñar Ciencias: desafíos, estrategias y oportunidades.

9. González, A. (2009). ABC en la educación científica. manos en la Masa. Taller Latinoamericano Evaluar proyectos ECBI. Bogotá: Universidad de los Andes. Brasil: Instituto Oswaldo Cruz - FIOCRUZ.

10. Grynszpan, D. (2009). La ciencia en tu escuela. Taller latinoamericano Evaluar proyectos ECBI. Bogotá: Universidad de los Andes.

11. Harlen, W., \& Allende, J. (2007). Informe del Grupo de Trabajo sobre Colaboración Internacional en la Evaluación de "Educación en Ciencias Basada en la Indagación". Chile: Universidad de Chile.

12. López, P. (2009). Educación en Ciencias Basada en Indagación (ECBI) - Academia Chilena de Ciencias. Taller Latinoamericano Evaluar Proyectos ECBI. Bogotá: Universidad de los Andes.

13. Meisel, J. (2007). Factores Críticos Institucionales que tienen relación con el ambiente de aprendizaje y las competencias ciudadanas de los estudiantes. Bogotá: Universidad de los Andes.

14. NRC. (2000). Inquiry and the national science education standards: a guide for teaching and learning. Washington. 
15. NSRC. (1997). Science for all children. Washington.

16. Olier, C., Duque, M., \& Tiberio, J. (2007). An Assessment Information System for a K-12 Hands-On Program: The Pequeños Científicos Case. International Conference on Engineering Education - ICEE.

17. Prince, M., \& Felder, R. (2006). Inductive teaching and learning methods definitions comparisons and research bases. Journal Engineer Education, 95(2), 123-138.

18. UNESCO. (2001). Science, technology and mathematics education for human development. Goa, India: Unesco. 